\title{
Faithful editing of a tomato-specific mRNA editing site in transgenic tobacco chloroplasts
}

\author{
DANIEL KARCHER, SABINE KAHLAU, and RALPH BOCK \\ Max-Planck-Institut für Molekulare Pflanzenphysiologie, D-14476 Potsdam-Golm, Germany
}

\begin{abstract}
RNA editing sites and their site-specific trans-acting recognition factors are thought to have coevolved. Hence, evolutionary loss of an editing site by a genomic mutation is normally followed by the loss of the specific recognition factor for this site, due to the absence of selective pressure for its maintenance. Here, we have tested this scenario for the only tomato-specific plastid RNA editing site. A single C-to-U editing site in the tomato rps12 gene is absent from the tobacco and nightshade plastid genomes, where the presence of a genomic T nucleotide obviates the need for editing of the rps12 mRNA. We have introduced the tomato editing site into the tobacco rps12 gene by plastid transformation and find that, surprisingly, this heterologous site is efficiently edited in the transplastomic plants. This suggests that the trans-acting recognition factor for the rps12 editing site has been maintained, presumably because it serves another function in tobacco plastids. Bioinformatics analyses identified an editing site in the rpoB gene of tobacco and tomato whose sequence context exhibits striking similarity to that of the tomato rps12 editing site. This may suggest that requirement for $r p o B$ editing resulted in maintenance of the rps 12 editing activity or, alternatively, the pre-existing $r p o B$ editing activity facilitated the evolution of a novel editing site in rps12.
\end{abstract}

Keywords: Nicotiana tabacum; Solanum lycopersicum; plastid; evolution; rps12; RNA editing; plastid transformation

\section{INTRODUCTION}

RNA editing is a post-transcriptional process changing the identity of individual nucleotides in a transcript. In higher plants, editing by pyrimidine conversions occurs in both DNA-containing cell organelles, the plastids, and the mitochondria. RNA editing in plastids of higher plants proceeds exclusively by cytidine-to-uridine conversions (for review, see Bock 2000, 2001a), whereas, in hornwort chloroplasts, also "reverse" editing by U-to-C transitions has been found (Yoshinaga et al. 1996; Kugita et al. 2003). Interestingly, the RNA editing processes in plant mitochondria and plastids exhibit striking similarities (for reviews, see Hanson et al. 1996; Bock 2001a), suggesting that they originate from common evolutionary roots.

The vast majority of known plastid RNA editing sites are located within coding regions and, with only very few exceptions (Hirose et al. 1996; Kudla and Bock 1999), editing alters the coding properties of the affected triplets.

Reprint requests to: Ralph Bock, Max-Planck-Institut für Molekulare Pflanzenphysiologie, Am Mühlenberg 1, D-14476 Potsdam-Golm, Germany; e-mail: rbock@mpimp-golm.mpg.de; fax: +49 (0)331 567-8701. Article published online ahead of print. Article and publication date are at http://www.rnajournal.org/cgi/doi/10.1261/rna.823508.
The changes brought about by mRNA editing usually result in the restoration of phylogenetically conserved amino acid residues (Maier et al. 1992a,b). Generation of transgenic plants with a noneditable version of a chloroplast gene has provided direct evidence for the functional significance of plastid mRNA editing (Bock et al. 1994).

RNA editing sites are generally believed to be recognized by the interplay between a cis-acting element at the RNA level and a proteinaceous trans-acting factor binding to the cis-element. The cis-acting element usually resides in the region immediately upstream of the editing site, as revealed by extensive analyses both in vivo and in vitro (Bock et al. 1996; Chaudhuri and Maliga 1996; Bock et al. 1997; Hermann and Bock 1999; Hirose and Sugiura 2001; Miyamoto et al. 2002; Hayes et al. 2006; Hayes and Hanson 2007). At least one site-specific trans-acting recognition factor is thought to bind to this cis-element (Chaudhuri et al. 1995; Bock and Koop 1997; Miyamoto et al. 2002, 2004). Recently, candidate site-specific editing factors have been identified for two RNA editing sites in the ndhD mRNA (Kotera et al. 2005; Okuda et al. 2006, 2007). The two proteins are members of the pentatricopeptide repeat (PPR) proteins, one of the largest protein families in plants, whose members appear to fulfill multifarious functions in plastid and mitochondrial gene expression. 
Interestingly, many of the known plastid RNA editing sites are only poorly conserved between species, indicating a remarkable evolutionary plasticity of the editing apparatus (Zeltz et al. 1993; Freyer et al. 1995, 1997; Fiebig et al. 2004). Editing sites can be easily lost by acquisition of a genomic C-to-T mutation, and loss of the editing site is usually followed by the loss of the site-specific trans-acting recognition factor, due to the absence of selective pressure for its maintenance. Evidence for this coevolution between editing sites and trans-acting specificity factors has been provided by in vitro studies (Miyamoto et al. 2002) and by plastid transformation experiments, in which heterologous editing sites were introduced into chloroplast genes: A site taken from species $\mathrm{A}$ and introduced into the plastid genome of species B usually cannot be edited, if species B lacks this site. For example, when the spinach $p s b F$ editing site was transplanted into the tobacco $p s b F$ gene (which naturally does not have the editing site), tobacco plastids were incapable of editing the spinach site (Bock et al. 1994; Bock and Koop 1997). Likewise, a heterologous maize editing site (from the $r p o B$ transcript) remained unedited when introduced into the tobacco plastid genome (Reed and Hanson 1997). The only exception reported to date is a site within the $n d h A$ gene of some but not all tobacco species: It is not present in the allotetraploid cultivated tobacco, Nicotiana tabacum, and one of its diploid progenitor species, Nicotiana sylvestris, but is present in the other diploid ancestor species, Nicotiana tomentosiformis (Schmitz-Linneweber et al. 2001). Nonetheless, N. tabacum and N. sylvestris possess the activity to edit the site (Tillich et al. 2006), possibly suggesting that, over short evolutionary distances, editing activities can be maintained for some time after the loss of the editing site.

We have recently conducted a comprehensive analysis of plastid RNA editing in Solanaceous plants (Kahlau et al. 2006) by comparing the editing patterns of three fully sequenced plastid genomes: tobacco (Nicotiana tabacum; Tsudzuki et al. 2001), deadly nightshade (Atropa belladonna; Schmitz-Linneweber et al. 2002), and tomato (Solanum lycopersicum; Kahlau et al. 2006). This comparison revealed the existence of a single tomato-specific editing site that was present neither in tobacco nor in Atropa. Editing in the second position of codon 74 of the rps12 transcript changes a genomically encoded UCA serine codon into a UUA leucine codon in tomato. In tobacco and Atropa, a TTA leucine codon is already present at the DNA level, obviating the need for RNA editing (Fig. 1).

To gain more information about the coevolution of plastid editing sites and their specific recognition factors, we have transplanted the rps12 editing site from the tomato into the tobacco rps12 gene. We report that, surprisingly, this heterologous site was faithfully edited in tobacco. We provide a possible mechanistic explanation for this finding, which may have general implications for the evolution of RNA editing sites in plant organelles.

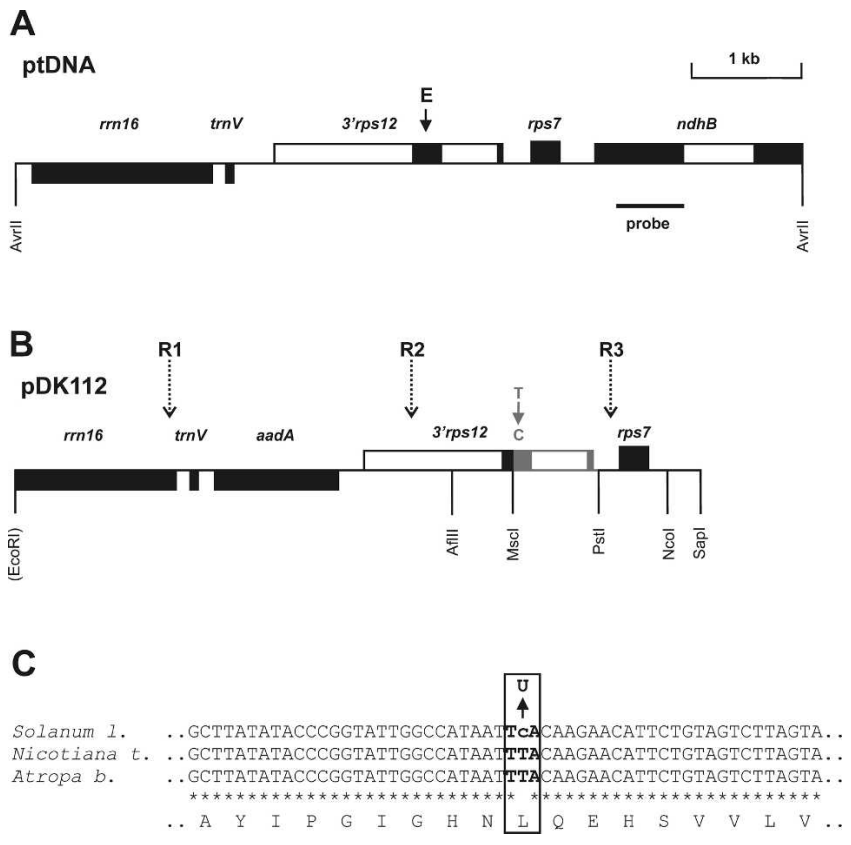

FIGURE 1. Construction of a plastid transformation vector to introduce the tomato rps12 editing site into the tobacco rps12 gene. $(A)$ Map of the rps12 region in the inverted repeat region of the tobacco plastid genome (ptDNA). Genes above the lines are transcribed from the left to the right; genes below the line are transcribed in the opposite direction. Location of the rps12 site edited in tomato is indicated by an arrowhead. The map depicts an AvrII fragment, the restriction enzyme employed for RFLP analysis (Fig. 2). The location of the RFLP probe is also indicated. $(B)$ Physical map of the plastid transformation vector ( $\mathrm{pDK} 112$ ) constructed to integrate the tomato rps12 editing site into the tobacco plastid genome by homologous recombination. The selectable marker gene for chloroplast transformation, aadA (Svab and Maliga 1993), was inserted into the intergenic spacer between the $3^{\prime}$ portion of $r p s 12$ and the $\operatorname{trn} V$ gene. Note that, due to the large distance between the aadA selection marker and the point mutation to be introduced, homologous recombination can be resolved in between the two genetic changes, resulting in transformants that harbor the aadA but lack the tomato rps12 editing site. Relevant restriction sites used for cloning are indicated; sites lost by ligation of heterologous ends are shown in parentheses. The tomato restriction fragment containing the editing site is shown in gray. It exchanged the corresponding tobacco fragment (MscI/PstI). The mutation in exon II creating the tomato-specific rps12 editing site by a T-to-C exchange is marked. Plastid transformation and selection for spectinomycin resistance produces two types of transplastomic lines: Homologous recombination at sites $\mathrm{R} 1$ and $\mathrm{R} 3$ results in integration of both the selectable marker gene aadA and the rps12 editing site, whereas recombination at sites $\mathrm{R} 1$ and $\mathrm{R} 2$ integrates only the aadA into the plastid genome, but not the editing site. $(C)$ Partial sequence alignment of the rps12 region containing the mRNA editing site in tomato. Sequences are shown for three Solanaceous species: tomato (Solanum l.), tobacco (Nicotiana t.), and deadly nightshade (Atropa b.). Note complete sequence identity with exception of the editing position in tomato (lowercase c). The codon changed from serine to leucine by RNA editing in tomato is boxed; the editing event is indicated by the $\mathrm{C}$-to- $\mathrm{U}$ conversion. The encoded amino acid sequence is shown in the one-letter code below the nucleotide sequence. 


\section{RESULTS AND DISCUSSION}

\section{Integration of the tomato rps12 editing site into the tobacco plastid genome}

The plastid rps12 gene encodes ribosomal protein S12, an essential component of the small subunit of the prokaryotic $70 S$ ribosome (Alksne et al. 1993). In higher plant plastids, the rps12 gene consists of three exons and exhibits an unusual split structure. Exon 1 is located in the large singlecopy region of the chloroplast genome, far away from exons 2 and 3, which are located in the inverted repeat region (Fig. 1A). Joining of exons 1 and 2 occurs by transsplicing which generates mature rps12 mRNA from two separately transcribed pieces (Zaita et al. 1987; Kohchi et al. 1988). The tomato-specific rps12 editing site (Kahlau et al. 2006) is located in exon 2 and represents the only nucleotide in which the tomato exon 2 sequence deviates from the corresponding tobacco sequence (Fig. 1; data not shown).

To introduce the tomato rps12 editing site into the tobacco rps12 gene, we transplanted the corresponding tomato sequence into a cloned tobacco plastid DNA fragment containing rps12 exons 2 and 3 (Fig. 1). To facilitate the selection of chloroplast transformants, the selectable marker gene aadA (Svab and Maliga 1993) was integrated into the adjacent intergenic spacer between the rps12-rps7 operon and the $\operatorname{trn} V$ gene (Fig. 1B). The rps12 exons 2 and 3 are part of a complex transcription unit (Koller et al. 1987; Zaita et al. 1987; Hildebrand et al. 1988), which precludes integration of the aadA marker in closer proximity to the editing site. Thus, in plastid transformation vector pDK112 (Fig. 1B), the mutation creating the editing site is $\sim 1.7 \mathrm{~kb}$ away from the selectable spectinomycin resistance gene aadA. This rather large distance between mutation and marker gene is expected to result in frequent resolution of homologous recombination in between the two genetic changes, thus separating the aadA marker from the mutation (Bock et al. 1994; Bock and Maliga 1995; Hager et al. 2002). Consequently, two different types of chloroplast transformants are expected to be obtained: (1) transformants carrying only the aadA but not the tomato rps12 editing site and (2) transformants having incorporated both the aadA gene and the mutation in $r p s 12$ (Fig. 1).

Biolistic bombardment of young leaves from aseptically grown tobacco plants with plasmid pDK112-coated gold particles was followed by selection of primary spectinomycinresistant cell lines. Successful chloroplast transformation was verified by tests for double resistance on medium containing two aminoglycoside antibiotics, spectinomycin, and streptomycin (Svab and Maliga 1993; Bock 2001b), and further confirmed by PCR assays using aadA genespecific primers (Bock et al. 1996; data not shown). Chloroplast transformants were purified to homoplasmy by passing them through additional regeneration cycles under antibiotic selection to eliminate residual copies of the wild-type chloroplast genome. Homoplasmy of the transplastomic lines was finally confirmed by RFLP analysis and transgene inheritance assays (Fig. 2A,B). Maternal inheritance of the antibiotic resistances confirmed transgene localization in the plastid genome; lack of segregation in the $\mathrm{T} 1$ generation (Fig. 2B) proved homoplasmy of the transplastomic lines.

Due to incomplete linkage between the selectable marker gene and the point mutation creating the tomato-specific editing site, the two expected types of plastid transformants were obtained. DNA sequencing of PCR products generated with rps12-specific primers revealed that 10 out of
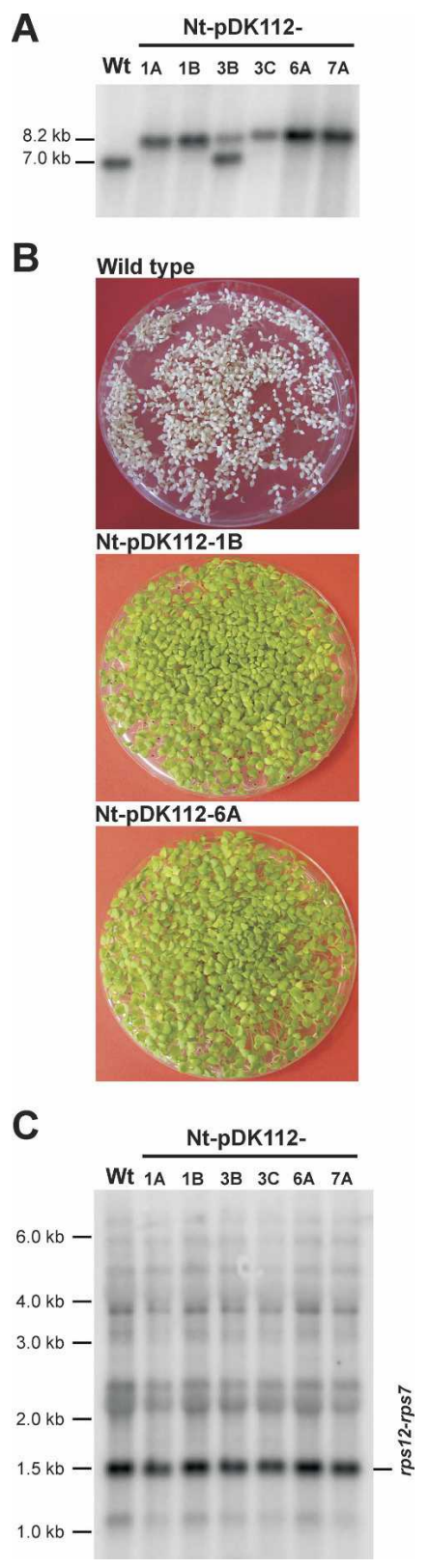

FIGURE 2. (Legend on next page) 
12 transformants carried only the aadA marker, but not the rps12 editing site. However, two transformants could be isolated that harbored also the point mutation in rps12, creating the tomato-specific editing site (lines Nt-pDK112$1 \mathrm{~B}$ and Nt-pDK112-3B; Fig. 2).

Interestingly, when still being heteroplasmic, the transplastomic lines with the rps12 mutation showed evidence of active gene conversion and/or recombination between wildtype plastid genomes and transgenic genomes. This was discovered by direct sequencing of PCR products covering the rps12 gene. The sequencing revealed a mixed population of transgenic plastid genomes which all carried the $\operatorname{aad} A$, but were heterogeneous with respect to the presence of the point mutation in rps12. Consequently, purification of these lines to homoplasmy and segregation of the two transgenic genome types allowed isolation of transplastomic lines with and without the rps12 mutation from one and the same primary transformed clone. For example, line Nt-pDK112-1B is homoplasmic for the tomato (editing site-containing) allele of $\operatorname{rps} 12$, whereas line Nt-pDK112-

FIGURE 2. Analysis of plastid transformants generated with vector pDK112. (A) Southern blot confirming plastid transformation and assessing homoplasmy of transplastomic lines. Digestion with the restriction enzyme AvrII produces an $\sim 7.0$-kb fragment in the wild type and an $\sim 8.2$-kb fragment in all transplastomic lines. This size difference corresponds to the size of the aadA cassette. Note that line $\mathrm{Nt}-\mathrm{pDK} 112-3 \mathrm{~B}$ is still heteroplasmic as evidenced by the presence of both the $7.0-\mathrm{kb}$ wild-type fragment and the $8.2-\mathrm{kb}$ fragment specific to the transplastome. This line was purified to homoplasmy by an additional regeneration round under antibiotic selection (not shown). (B) Confirmation of homoplasmy of transplastomic lines by seed assays and lack of a mutant phenotype in tobacco plants carrying the tomato-specific rps12 mRNA editing site. Seeds from the wild-type and the selfed transplastomic lines Nt-pDK112-1B and Nt-pDK112$6 \mathrm{~A}$ were germinated on medium with spectinomycin $(500 \mathrm{mg} / \mathrm{L})$. The progeny from the two transplastomic lines shown here is uniformly resistant to spectinomycin demonstrating complete absence of wildtype genome copies (Svab and Maliga 1993; Bock 2001a,b). As a control, untransformed wild-type seedlings are shown which are sensitive to spectinomycin and bleach out rapidly after germination. Transplastomic line Nt-pDK112-1B carries both the aadA selection marker and the tomato rps12 editing site, whereas line Nt-pDK112-6A harbors only the aadA, but lacks the tomato editing site and thus has the unchanged wild-type tobacco rps12 sequence. Plants from the two lines are phenotypically identical also at later developmental stages (not shown), indicating that introduction of the tomato editing site into the tobacco plastid genome does not result in a mutant phenotype. $(C)$ Northern blot to analyze rps12 transcript pattern and RNA accumulation in transplastomic tobacco lines. Total cellular RNA was electrophoresed under denaturing conditions and hybridized to a radiolabeled PCR product covering all three exons of rps 12. In addition to the $\sim 1.5$-kb fully processed dicistronic rps12-rps7 mRNA, the probe recognizes several other RNA species which represent incompletely spliced transcripts and other unprocessed or partially processed precursor transcripts. mRNA accumulation and transcript patterns are indistinguishable between the wild-type and the Nt-pDK112 transplastomic plants, indicating that neither integration of the selectable marker gene aadA upstream of the rps12-rps7 operon (Fig. 1) nor introduction of the point mutation creating the rps12 editing site (in lines Nt-pDK112-1B and Nt-pDK112-3B) affect transcription and/or mRNA accumulation.
$1 \mathrm{~A}$ is homoplasmic for the tobacco rps12 allele lacking the editing site. Both lines stem from one and the same transformation event (primary transformant Nt-pDK1121). Similarly, line Nt-pDK112-3B has the editing site and line Nt-pDK112-3A does not. Gene conversion between a transplastome with the aadA and the tomato editing site and a wild-type genome provides a likely explanation for obtaining these two transplastomic genome types and is in line with the earlier finding that gene conversion operates at high activity in plastids (Khakhlova and Bock 2006).

\section{Analysis of tobacco plastid transformants carrying the tomato-specific rps12 editing site}

As the rps12 exons 2 and 3 are part of an operon which is transcribed as a polycistronic mRNA, it was important to verify that neither insertion of the aadA selection marker nor the point mutation introduced into exon 2 affect transcription of the operon or stability of the rps 12 mRNAs. We, therefore, comparatively analyzed accumulation of rps12 transcripts in wild-type and transplastomic tobacco lines (Fig. 2C). No significant difference was found in transcript pattern and mRNA accumulation levels between the wild type, the transplastomic lines only having the aadA marker and those additionally carrying the rps12 point mutation indicating that integration of the aadA and introduction of the editing site do not negatively affect synthesis or stability of rps12 transcripts.

Homoplasmic transplastomic tobacco plants with the tomato editing site in rps12 displayed no discernable phenotype when grown in in vitro culture or under greenhouse conditions (Fig. 2B; data not shown). This tentatively suggested that either the tomato site can be edited in tobacco or the site remains unedited and this is functionally irrelevant in that the S12 protein can function with either serine or leucine in the amino acid position corresponding to the codon containing the editing site.

\section{RNA editing of a tomato-specific editing site in transplastomic tobacco}

We next wanted to determine whether the tomato rps12 editing site can be processed by the tobacco editing machinery. To this end, we synthesized cDNA and directly sequenced the amplified cDNA population. As a control, the corresponding DNA amplification products were also sequenced. Surprisingly, the tomato-specific RNA editing site turned out to be fully edited in transplastomic tobacco (Fig. 3), indicating that tobacco plants possess the capacity to edit this foreign site. This suggests that the site-specific trans-acting recognition factor required for rps12 editing is present in tobacco chloroplasts, even though the editing site does not exist in tobacco.

Maintenance of the editing capacity in the absence of the target site can be fortuitous in that, despite the loss of the 


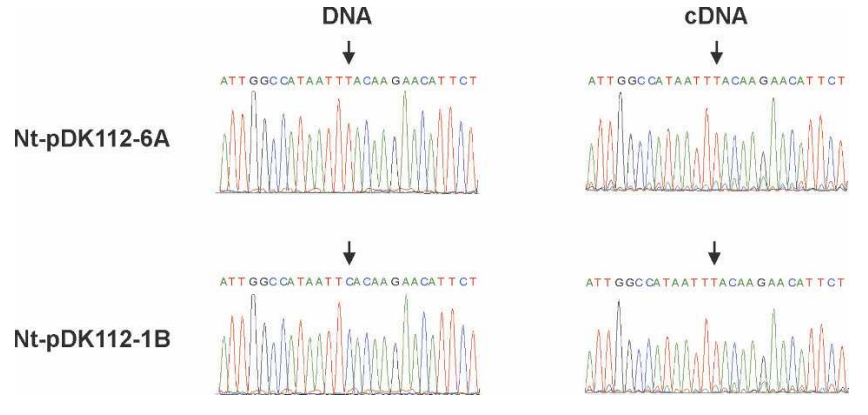

FIGURE 3. Analysis of mRNA editing in transplastomic tobacco plants. rps12 DNA and cDNA sequences are shown for a transplastomic tobacco plant harboring the tomato-specific rps12 editing site (Nt-pDK112-1B) and a control transplastomic line containing only the aadA selectable marker gene, but the wild-type tobacco sequence in rps12 (Nt-pDK112-6A). The RNA editing site is marked by vertical arrows in all sequences. Note complete C-to- $\mathrm{U}$ conversion in the cDNA sequence of line Nt-pDK112-1B suggesting efficient recognition of the heterologous tomato-specific editing site in transplastomic tobacco plants.

editing site in tobacco, the nuclear gene for the site-specific editing factor remained functional and escaped evolutionary deterioration. Alternatively, and perhaps more likely, the gene could be maintained by selection, because rps12 editing is not the only function of this trans-acting editing factor. Based on competition analysis in plants overexpressing individual editing sites, it has been suggested that some trans-acting editing factors may be involved in the editing of more than one site (Chateigner-Boutin and Hanson 2002). We, therefore, searched for possible alternative targets of the rps12 editing factor by analyzing all editing sites in the tobacco and tomato plastid genomes for sequence similarity to the tomato rps12 site. As earlier work had established that the essential cis-acting elements for editing site recognition reside in the region immediately upstream of the edited nucleotide (Bock et al. 1996; Chaudhuri and Maliga 1996; Bock et al. 1997; Hirose and Sugiura 2001; Miyamoto et al. 2002; Hayes et al. 2006; Hayes and Hanson 2007), we analyzed the sequence stretches ranging from position -31 nt upstream of the editing site to position +1 downstream. We used phylogenetic tree construction software to cluster all editing sites in the genome according to their sequence similarities (Fig. 4A). This analysis revealed a single editing site that clustered with the rps12 site: the editing site in codon 667 of $r p o B$, the gene encoding the catalytic $\beta$-subunit of the Escherichia coli-like plastid RNA polymerase (PEP). Alignment of the two sequences confirmed their high similarity (Fig. 4B) and may suggest that, indeed, the two sites are served by the same trans-acting specificity factor.

Earlier work has established that introduction into the plastid genome of extra copies of an editing site can result in reduced editing efficiency at both the endogenous and the transgenic editing site, presumably due to competition for trans-acting specificity factors that are present in limiting amounts (Chaudhuri et al. 1995; Chaudhuri and Maliga 1996). To determine whether or not the presence of an additional target site in rps12 makes the trans-acting site-specific factor limiting for $r p o B$ editing in tobacco, we also investigated the efficiency of $r p o B$ editing in codon 667. When the editing efficiency was determined by directly sequencing the amplified $r p o B$ cDNA populations from transplastomic plants harboring the rps12 site and control lines carrying only the aadA marker gene, the rpoB mRNA turned out to be fully edited at the site in codon 667 (data

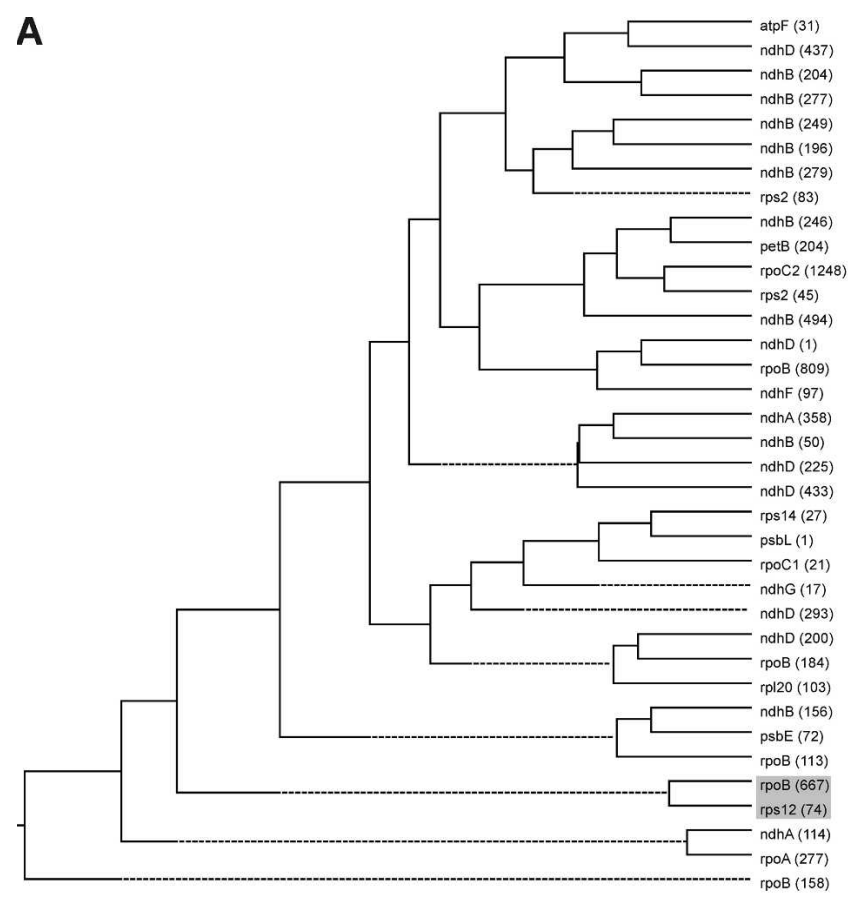

B

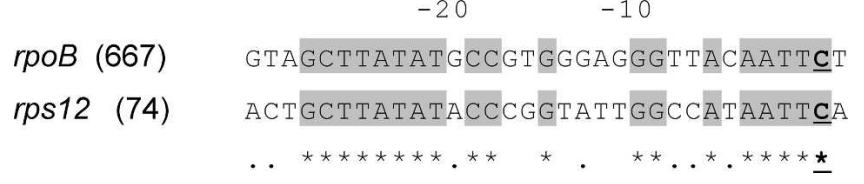

FIGURE 4. Identification of an editing site in the $r p o B$ gene which displays strong sequence similarity to the tomato $r p s 12$ site. $(A)$ Assessment of sequence similarities between editing sites using phylogenetic tree construction software. For all editing sites in the tomato plastid genome (Kahlau et al. 2006), the DNA sequence from position -31 to position +1 downstream from the editing site was used for tree construction. Only a single RNA editing site (located in codon 667 of the $r p o B$ mRNA) exhibits close sequence relatedness with the tomato-specific rps12 editing site (sites shaded) introduced into the tobacco plastid genome. Dotted lines indicate negative branch lengths. (B) Alignment of the regions immediately upstream of the tomato-specific rps12 editing site and the rpoB editing site in codon 667 of tobacco and tomato. Nucleotides identical in both sequences are indicated by asterisks and shading; positions in which both sequences differ only in the purine (A or $\mathrm{G}$ ) or the pyrimidine ( $\mathrm{T}$ or $\mathrm{C}$ ) are indicated by dots. The edited $\mathrm{C}$ is in bold and underlined. 
not shown). This, together with complete editing at the heterologous rps12 site (Fig. 3), indicates that the transacting editing factor(s) are not present in limiting amounts.

The tree construction approach taken here to identify sequence similarities between editing sites may serve as a useful tool to (1) detect editing sites with shared cis-acting recognition elements which may be served by identical trans-acting specificity factors and (2) predict editability of heterologous mRNA editing sites in plastid (and mitochondrial) genomes.

\section{Evolutionary considerations}

The editing of the heterologous rps12 editing site from tomato in transplastomic tobacco plants and the observed high similarity of the sequences upstream of the rps12 site and the $r p o B$ site in codon 667 raise two possible evolutionary scenarios. In the "editing early" scenario, rps12 editing could be the ancestral situation in Solanaceous plants and, during diversification of this family, the site was lost in multiple lineages, including the Atropa and tobacco clades. The editing activity for the rps12 site, however, was maintained, because the same trans-acting specificity factor is also required for editing of a site in $r p o B$ (which is present in all Solanaceae; Kahlau et al. 2006). In the alternative "editing late" scenario, rps12 editing represents a more recent acquisition and is newly evolved in the Solanum clade. The birth of this novel site may have been facilitated by the possibility to co-opt a preexisting editing activity, which had evolved earlier to edit a site in $r p o B$. Preexistence of the editing activity would make a genomic T-to-C mutation in rps12 codon 74 neutral, because it can immediately be corrected at the RNA level by editing (Covello and Gray 1993).

To distinguish between these two possibilities, we have conducted a preliminary phylogenetic analysis of rps12 editing. Our results suggest that the editing site is not unique to tomato and is present also in other Solanaceous species, for example, Petunia hybrida, Capsicum annuum, and Physalis alkekengi (data not shown). More importantly, the site is also present in species outside the Solanaceae family, for example, in Antirrhinum majus, a species in the Scrophulariacea, the sister family of Solanaceae, and in Buddleja davidii, a species in the family Buddlejaceae (data not shown). This may suggest that the "editing early" scenario represents the more likely evolutionary scenario for this site.

\section{MATERIALS AND METHODS}

\section{Plant material}

Tobacco plants (Nicotiana tabacum cv. Petit Havana) were grown under aseptic conditions on agar-solidified MS medium containing $30 \mathrm{~g} / \mathrm{L}$ sucrose (Murashige and Skoog 1962). Transplastomic lines were rooted and propagated on the same medium in the presence of spectinomycin $(500 \mathrm{mg} / \mathrm{L})$.

\section{Construction of chloroplast transformation vector pDK112}

The PstI site in cloning vector pMCS5 (MoBiTec) was deleted by digestion with AvrII and $\mathrm{XbaI}$ and subsequent relegation generating plasmid pDK108. The rps12-trnV region of the tobacco plastid genome was cloned as a 2337 base-pair (bp) SacII/NcoI restriction fragment into the similarly cut pDK108, resulting in plasmid pDK109. A 770-bp MscI/PstI fragment including the tomato-specific rps12 RNA editing site (Fig. 1B) was isolated from a cloned tomato plastid DNA fragment and replaced the corresponding fragment in pDK109, generating clone pDK110. Part of the vector backbone in plastid transformation vector pPRV111A (Zoubenko et al. 1994) was replaced with the corresponding region of pBluescript SK by ligation of two PvuII fragments, resulting in plasmid pDK111. Plasmid pDK110 was digested with AflII and SapI and ligated into the similarly cut vector pDK111, resulting in final transformation vector pDK112.

\section{Plastid transformation and selection of transplastomic tobacco lines}

Young leaves harvested form sterile tobacco plants were bombarded with plasmid pDK112-coated gold particles using the BioRad PDS1000He biolistic gun. Primary spectinomycin-resistant cell lines were selected on plant regeneration medium containing $500 \mathrm{mg} / \mathrm{L}$ spectinomycin dihydrochloride (Svab and Maliga 1993). Spontaneous spectinomycin-resistant plants were eliminated by double selection on medium containing spectinomycin and streptomycin (500 mg/L each; Svab and Maliga 1993; Bock 2001b). Putative plastid transformants were subjected to three additional rounds of regeneration on regeneration medium with spectinomycin to eliminate residual wild-type genome copies. Shoots resulting from the final round of regeneration were rooted on phytohormone-free spectinomycin-containing medium, transferred to soil, and further cultivated in the greenhouse.

For inheritance assays, surface-sterilized seeds were germinated on MS medium with spectinomycin (500 mg/L) and analyzed for uniparental inheritance of the aadA resistance gene used to introduce the tomato rps12 editing site into the tobacco plastid genome. Selfed transformants and crosses with a plastid transformant as the maternal parent give rise to resistant (green) progeny, whereas seeds collected from wild-type plants yield sensitive (white) seedlings.

\section{Isolation of nucleic acids}

Total plant DNA was isolated from fresh leaf tissue samples by a cetyltrimethylammoniumbromide (CTAB)-based method (Doyle and Doyle 1990). Total cellular RNA was extracted with the TriFast reagent (Peqlab GmbH). For reverse transcription, RNA samples were treated with DNase I (Roche Diagnostics $\mathrm{GmbH}$ ) to eliminate residual contaminating DNA.

\section{DNA and RNA gel blot analyses}

Total cellular DNA from wild-type and transplastomic plants was digested with restriction enzymes, separated in $0.8 \%$ agarose gels 
and transferred onto Hybond nylon membranes (Amersham) by capillary blotting. A PCR product obtained with primers $\mathrm{Nb0}\left(5^{\prime}-\right.$ TGCTTCTCTTCGATGGAAG-3') and P13 (5'-TTTTGGATCCTA TACATTTGTGTATTGATA-3') was radiolabeled with ${ }^{32} \mathrm{P}$-dCTP and used as a probe in Southern blot analyses (Fig. 1A). Likewise, a radiolabeled PCR product served as a specific probe in Northern blot analyses to specifically detect $r p s 12$ transcripts. The PCR product covers all three exons of rps 12 and was obtained with primer pair P184 (5'-CGGTTAGGATCAATCTAAACCAACCC-3') and P185 (5'-GAGCGTGAAAGGGGTTCAAGAATC-3') using cDNA as a template. Hybridizations were performed at $65^{\circ} \mathrm{C}$ in Rapid-Hyb buffer (Amersham) following the manufacturer's protocol.

\section{cDNA synthesis and PCR}

RNA samples treated with RNase-free DNase I were reversetranscribed with SuperScript III reverse transcriptase (Invitrogen) following the instructions of the supplier. cDNA synthesis was primed with random hexanucleotide primers. cDNA samples were amplified with the rps12-specific primer pair P184/P185 in an Eppendorf thermocycler using Taq DNA polymerase (Promega) and standard protocols. The genomic rps12 sequence was amplified with primers PSeq-rps12 (5'-GGGTGACTTATCTGTCAAC$\left.3^{\prime}\right)$ and PRps12-rev (5'-ATGGTAGCCTGCTCCAGTC-3'). DNA and cDNA amplification products were purified for sequencing by electrophoresis in $1.5 \%$ agarose gels and subsequent extraction from gel slices using the Nucleospin Extract II kit (MachereyNagel). Direct sequencing of amplification products was performed by cycle sequencing. Oligonucleotide P184 served as sequencing primer for cDNA PCR products; DNA amplification products were sequenced with primer PSeq-rps12.

\section{Bioinformatics analyses}

Sequence similarities between putative cis-acting recognition elements upstream of plastid RNA editing sites were identified using phylogenetic tree construction software (MegAlign program from the Lasergene software package, DNASTAR; GATC Biotech). For each editing site, a 33-bp sequence stretch was entered, including the editing site itself, 31 bp upstream and 1 bp downstream. As the upstream cis-elements for editing site recognition can be somewhat variable in their distance from the edited $\mathrm{C}$, the unaligned raw sequences were used for tree construction. This procedure ensured that all sequences had the edited $\mathrm{C}$ in the same position and no gaps were introduced through the alignment algorithm. The length of each pair of branches represents the distance between sequence pairs. Dotted lines in the phenogram indicate negative branch lengths, a common consequence of averaging. The phenogram with balanced branches averages the distances between ancestors in the tree.

\section{ACKNOWLEDGMENTS}

We thank the MPI-MP Green Team for plant care and cultivation, Anne Schadach and Fei Zhou for technical assistance, and Dr. Stephanie Ruf for help with chloroplast transformation. This research was supported by a grant from the European Union (FP6 Plastomics project LSHG-CT-2003-503238) to R.B. and by the Max Planck Society.

Received September 11, 2007; accepted October 29, 2007.

\section{REFERENCES}

Alksne, L.E., Anthony, R.A., Liebman, S.W., and Warner, J.R. 1993. An accuracy center in the ribosome conserved over 2 billion years. Proc. Natl. Acad. Sci. 90: 9538-9541.

Bock, R. 2000. Sense from nonsense: How the genetic information of chloroplasts is altered by RNA editing. Biochimie 82: 549-557.

Bock, R. 2001a. RNA editing in plant mitochondria and chloroplasts. In Frontiers in molecular biology: RNA editing (ed. B.L. Bass), pp. 38-60. Oxford University Press, New York.

Bock, R. 2001b. Transgenic chloroplasts in basic research and plant biotechnology. J. Mol. Biol. 312: 425-438.

Bock, R. and Koop, H.-U. 1997. Extraplastidic site-specific factors mediate RNA editing in chloroplasts. EMBO J. 16: 3282-3288.

Bock, R. and Maliga, P. 1995. In vivo testing of a tobacco plastid DNA segment for guide RNA function in psbL editing. Mol. Gen. Genet. 247: 439-443.

Bock, R., Kössel, H., and Maliga, P. 1994. Introduction of a heterologous editing site into the tobacco plastid genome: The lack of RNA editing leads to a mutant phenotype. $E M B O ~ J .13$ : 4623-4628.

Bock, R., Hermann, M., and Kössel, H. 1996. In vivo dissection of cisacting determinants for plastid RNA editing. EMBO J. 15: 5052-5059.

Bock, R., Hermann, M., and Fuchs, M. 1997. Identification of critical nucleotide positions for plastid RNA editing site recognition. RNA 3: 1194-1200.

Chateigner-Boutin, A.-L. and Hanson, M.R. 2002. Cross-competition in transgenic chloroplasts expressing single editing sites reveals shared cis elements. Mol. Cell. Biol. 22: 8448-8456.

Chaudhuri, S. and Maliga, P. 1996. Sequences directing C to U editing of the plastid psbL mRNA are located within a 22 nucleotide segment spanning the editing site. EMBO J. 15: 5958-5964.

Chaudhuri, S., Carrer, H., and Maliga, P. 1995. Site-specific factor involved in the editing of the psbL mRNA in tobacco plastids. EMBO J. 14: 2951-2957.

Covello, P.S. and Gray, M.W. 1993. On the evolution of RNA editing. Trends Genet. 9: 265-268.

Doyle, J.J. and Doyle, J.L. 1990. Isolation of plant DNA from fresh tissue. Focus 12: 13-15.

Fiebig, A., Stegemann, S., and Bock, R. 2004. Rapid evolution of RNA editing sites in a small non-essential plastid gene. Nucleic Acids Res. 32: 3615-3622. doi: 10.1093/nar/gkh695.

Freyer, R., Lopez, C., Maier, R.M., Martin, M., Sabater, B., and Kössel, H. 1995. Editing of the chloroplast $n d h B$ encoded transcript shows divergence between closely related members of the grass family (Poaceae). Plant Mol. Biol. 29: 679-684.

Freyer, R., Kiefer-Meyer, M.-C., and Kössel, H. 1997. Occurrence of plastid RNA editing in all major lineages of land plants. Proc. Natl. Acad. Sci. 94: 6285-6290.

Hager, M., Hermann, M., Biehler, K., Krieger-Liszkay, A., and Bock, R. 2002. Lack of the small plastid-encoded PsbJ polypeptide results in a defective water-splitting apparatus of photosystem II, reduced photosystem I levels, and hypersensitivity to light. J. Biol. Chem. 277: 14031-14039.

Hanson, M.R., Sutton, C.A., and Lu, B. 1996. Plant organelle gene expression: Altered by RNA editing. Trends Plant Sci. 1: 57-64.

Hayes, M.L. and Hanson, M.R. 2007. Identification of a sequence motif critical for editing of a tobacco chloroplast transcript. RNA 13: $281-288$.

Hayes, M.L., Reed, M.L., Hegeman, C.E., and Hanson, M.R. 2006. Sequence elements critical for efficient RNA editing of a tobacco chloroplast transcript in vivo and in vitro. Nucleic Acids Res. 34: 3742-3754. doi: 10.1093/nar/gkl490.

Hermann, M. and Bock, R. 1999. Transfer of plastid RNA-editing activity to novel sites suggests a critical role for spacing in editingsite recognition. Proc. Natl. Acad. Sci. 96: 4856-4861.

Hildebrand, M., Hallick, R.B., Passavant, C.W., and Bourque, D.P. 1988. Trans-splicing in chloroplasts: The rps12 loci of Nicotiana tabacum. Proc. Natl. Acad. Sci. 85: 372-376. 
Hirose, T. and Sugiura, M. 2001. Involvement of a site-specific transacting factor and a common RNA-binding protein in the editing of chloroplast mRNAs: Development of a chloroplast in vitro RNA editing system. EMBO J. 20: 1144-1152.

Hirose, T., Fan, H., Suzuki, J.Y., Wakasugi, T., Tsudzuki, T., Kössel, H., and Sugiura, M. 1996. Occurrence of silent RNA editing in chloroplasts: Its species specificity and the influence of environmental and developmental conditions. Plant Mol. Biol. 30: 667-672.

Kahlau, S., Aspinall, S., Gray, J.C., and Bock, R. 2006. Sequence of the tomato chloroplast DNA and evolutionary comparison of solanaceous plastid genomes. J. Mol. Evol. 63: 194-207.

Khakhlova, O. and Bock, R. 2006. Elimination of deleterious mutations in plastid genomes by gene conversion. Plant J. 46: 85-94.

Kohchi, T., Umesono, K., Ogura, Y., Komine, Y., Nakahigashi, K., Komano, T., Yamada, Y., Ozeki, H., and Ohyama, K. 1988. A nicked group II intron and trans-splicing in liverwort, Machantia polymorpha, chloroplasts. Nucleic Acids Res. 16: 10025-10036. doi: 10.1093/nar/16.21.10025.

Koller, B., Fromm, H., Galun, E., and Edelman, M. 1987. Evidence for in vivo trans splicing of pre-mRNAs in tobaco chloroplasts. Cell 48: 111-119.

Kotera, E., Tasaka, M., and Shikanai, T. 2005. A pentatricopeptide repeat protein is essential for RNA editing in chloroplasts. Nature 433: 326-330.

Kudla, J. and Bock, R. 1999. RNA editing in an untranslated region of the Ginkgo chloroplast genome. Gene 234: 81-86.

Kugita, M., Yamamoto, Y., Fujikawa, T., Matsumoto, T., and Yoshinaga, K. 2003. RNA editing in hornwort chloroplasts makes more than half the genes functional. Nucleic Acids Res. 31: 24172423. doi: $10.1093 /$ nar/gkg327.

Maier, R.M., Hoch, B., Zeltz, P., and Kössel, H. 1992a. Internal editing of the maize chloroplast ndhA transcript restores codons for conserved amino acids. Plant Cell 4: 609-616.

Maier, R.M., Neckermann, K., Hoch, B., Akhmedov, N.B., and Kössel, H. 1992b. Identification of editing positions in the $n d h B$ transcript from maize chloroplasts reveals sequence similarities between editing sites of chloroplasts and plant mitochondria. Nucleic Acids Res. 20: 6189-6194. doi: 10.1093/nar/20.23.6189.

Miyamoto, T., Obokata, J., and Sugiura, M. 2002. Recognition of RNA editing sites is directed by unique proteins in chloroplasts: Biomedical identification of cis-acting elements and trans-acting factors involved in RNA editing in tobacco and pea chloroplasts. Mol. Cell. Biol. 22: 6726-6734.

Miyamoto, T., Obokata, J., and Sugiura, M. 2004. A site-specific factor interacts directly with its cognate RNA editing site in chloroplast transcripts. Proc. Natl. Acad. Sci. 101: 48-52.
Murashige, T. and Skoog, F. 1962. A revised medium for rapid growth and bio assays with tobacco tissue culture. Physiol. Plant. 15: 473-497.

Okuda, K., Nakamura, T., Sugita, M., Shimizu, T., and Shikanai, T. 2006. A pentatricopeptide repeat protein is a site recognition factor in chloroplast RNA editing. J. Biol. Chem. 281: 37661-37667.

Okuda, K., Myouga, F., Motohashi, R., Shinozaki, K., and Shikanai, T. 2007. Conserved domain structure of pentatricopeptide repeat proteins involved in chloroplast RNA editing. Proc. Natl. Acad. Sci. 104: $8178-8183$.

Reed, M.L. and Hanson, M.R. 1997. A heterologous maize rpoB editing site is recognized by transgenic tobacco chloroplasts. Mol. Cell. Biol. 17: 6948-6952.

Schmitz-Linneweber, C., Tillich, M., Herrmann, R.G., and Maier, R.M. 2001. Heterologous, splicing-dependent RNA editing in chloroplasts: Allotetraploidy provides trans-factors. EMBO J. 20: $4874-4883$.

Schmitz-Linneweber, C., Regel, R., Du, T.G., Hupfer, H., Herrmann, R.G., and Maier, R.M. 2002. The plastid chromosome of Atropa belladonna and its comparison with that of Nicotiana tabacum: The role of RNA editing in generating divergence in the process of plant speciation. Mol. Biol. Evol. 19: 1602-1612.

Svab, Z. and Maliga, P. 1993. High-frequency plastid transformation in tobacco by selection for a chimeric aadA gene. Proc. Natl. Acad. Sci. 90: 913-917.

Tillich, M., Poltnigg, P., Kushnir, S., and Schmitz-Linneweber, C. 2006. Maintenance of plastid RNA editing activities independently of their target sites. EMBO Rep. 7: 308-313.

Tsudzuki, T., Wakasugi, T., and Sugiura, M. 2001. Comparative analysis of RNA editing sites in higher plant chloroplasts. J. Mol. Evol. 53: 327-332.

Yoshinaga, K., Iinuma, H., Masuzawa, T., and Ueda, K. 1996. Extensive RNA editing of $\mathrm{U}$ to $\mathrm{C}$ in addition to $\mathrm{C}$ to $\mathrm{U}$ substitution in the rbcL transcripts of hornwort chloroplasts and the origin of RNA editing in green plants. Nucleic Acids Res. 24: 1008-1014. doi: 10.1093/nar/24.6.1008.

Zaita, N., Torazawa, K., Shinozaki, K., and Sugiura, M. 1987. Transsplicing in vivo: Joining of transcripts from the "divided" gene for ribosomal protein S12 in the chloroplasts of tobacco. FEBS Lett. 210: $153-156$.

Zeltz, P., Hess, W.R., Neckermann, K., Börner, T., and Kössel, H. 1993. Editing of the chloroplast rpoB transcript is independent of chloroplast translation and shows different patterns in barley and maize. EMBO J. 12: 4291-4296.

Zoubenko, O.V., Allison, L.A., Svab, Z., and Maliga, P. 1994. Efficient targeting of foreign genes into the tobacco plastid genome. Nucleic Acids Res. 22: 3819-3824. doi: 10.1093/nar/22.19.3819. 

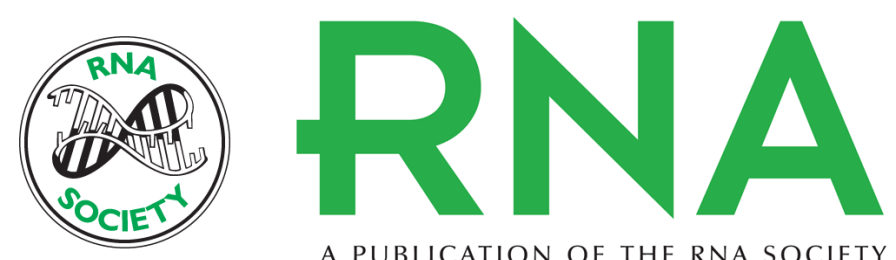

A PUBLICATION OF THE RNA SOCIETY

\section{Faithful editing of a tomato-specific mRNA editing site in transgenic tobacco chloroplasts}

Daniel Karcher, Sabine Kahlau and Ralph Bock

RNA 2008 14: 217-224

References This article cites 48 articles, 18 of which can be accessed free at:

http://rnajournal.cshlp.org/content/14/2/217.full.html\#ref-list-1

\section{License}

Email Alerting Service

Receive free email alerts when new articles cite this article - sign up in the box at the top right corner of the article or click here. 